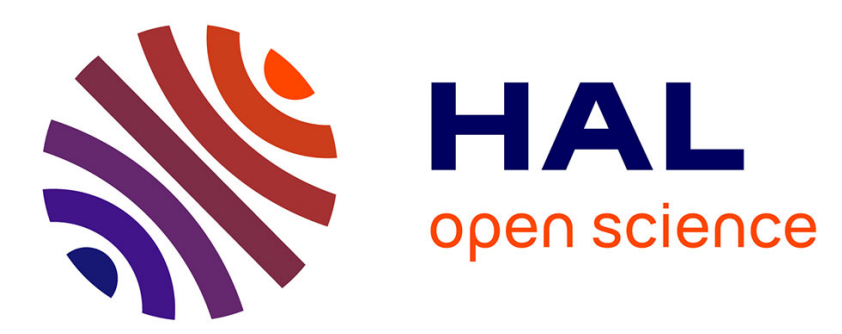

\title{
Tenir un cahier dans la région cotonnière du Mali. Support d'écriture et rapport à soi
}

\author{
Aïssatou Mbodj-Pouye
}

\section{To cite this version:}

Aïssatou Mbodj-Pouye. Tenir un cahier dans la région cotonnière du Mali. Support d'écriture et rapport à soi. Annales. Histoire, Sciences sociales, 2009, 2009-4, pp.855-885. halshs-00695854

\section{HAL Id: halshs-00695854 \\ https://shs.hal.science/halshs-00695854}

Submitted on 10 May 2012

HAL is a multi-disciplinary open access archive for the deposit and dissemination of scientific research documents, whether they are published or not. The documents may come from teaching and research institutions in France or abroad, or from public or private research centers.
L'archive ouverte pluridisciplinaire HAL, est destinée au dépôt et à la diffusion de documents scientifiques de niveau recherche, publiés ou non, émanant des établissements d'enseignement et de recherche français ou étrangers, des laboratoires publics ou privés. 


\section{Tenir un cahier dans la région cotonnière du Mali}

\section{Support d'écriture et rapport à soi*}

Loin des résultats attendus d'une alphabétisation fonctionnelle destinée à améliorer production agricole et organisation communautaire, disposer d'un cahier à soi est un des avantages que certains agriculteurs de la région cotonnière du Mali retirent de l'alphabétisation. Ces cahiers, du format d'un cahier d'écolier, ont un contenu très hétérogène, qui va du relevé de dates de naissance à la tenue d'une comptabilité, de la notation de titres de chansons entendues à la radio à la transcription de formules magiques, de la copie de prières à la consignation de résultats sportifs. Trois langues (français, bambara et arabe) et deux graphies (latine et arabe) s'y mêlent, inégalement maîtrisées par des scripteurs aux trajectoires diverses.

Consigner sur un même support des notes variées, et constituer dans la durée un ordre, même fragmentaire, à travers la régularité de cette pratique, est un geste dont les études sur la culture écrite en Occident fournissent une série d'exemples, notamment les livres de raison de l'Europe moderne. Cette comparaison ouvre une première interrogation autour de la sphère recouverte par ces écrits, personnelle, familiale ou domestique. Ses contours sont-ils déjà dessinés dans la société rurale du Mali contemporain où cette pratique s'observe? L'hypothèse selon laquelle l'émergence de nouvelles pratiques de l'écrit accompagne un processus plus large de privatisation, classique dans les travaux sur l'histoire de l'alphabétisation occidentale, demande à être mise à l'épreuve sur ce nouveau terrain ${ }^{1}$.

\footnotetext{
* Mes remerciements s'adressent d'abord à mes interlocuteurs au Mali qui ont eu la gentillesse de me montrer leurs écrits, d'en discuter avec moi et de me permettre de les photographier. Mes collègues de l'équipe Anthropologie de l'écriture du IIAC (CNRS-EHESS) ont été de précieux relecteurs de ce texte, merci en particulier à Claire Bustarret et Béatrice Fraenkel. Le travail mené en commun avec Cécile Van den Avenne nourrit la description de ces écrits, notamment dans ses aspects linguistiques.

${ }^{1}$ Roger ChARTIER, «Les pratiques de l'écrit », in Philippe ARIES et Georges DUBy (dir.), Histoire de la vie privée, tome 3, De la Renaissance aux Lumières vol. dir. par Roger CHARTIER, Paris, Le Seuil, 1986, p. 113-161.
} 
La notion de privé s'articule à deux autres, celle de l'individualisation et celle du rapport à soi, au sein d'un questionnement sur les modes de subjectivation par l'écrit qui constitue une ligne d'investigation privilégiée des travaux sur la culture écrite ${ }^{2}$. On sait que l'écrit joue un rôle central dans la manière dont le pouvoir s'applique à des populations et à individus depuis l'époque coloniale ${ }^{3}$, suscitant une gamme de réactions, de la résistance à l'appropriation. La notion de rapport à soi invite à explorer la manière dont certains individus se ressaisissent de ces dispositifs à leurs propres fins. La question qui nous occupera ici s'inscrit dans cette perspective : en quoi la tenue d'un cahier personnel, ouvrant un espace à soi dans le contexte d'une culture de l'écrit tramée d'injonctions fortes, permet-elle d'expérimenter de nouveaux rapports à soi ?

L'ouvrage récemment dirigé par Karin Barber sur les usages de l'écrit dans l'Afrique coloniale et postcoloniale montre que l'imposition de l'écrit en graphie latine a très tôt fait l'objet de reprises locales, sous impulsion missionnaire notamment ${ }^{4}$. La plupart des études de cas que réunit ce livre portent sur des individus que leurs pratiques d'écriture et leur statut de clerc singularisent au sein de leur environnement immédiat. Les pratiques analysées ici se situent en deçà de ces profils intermédiaires entre des populations peu alphabétisées et des élites lettrées. Elles témoignent de l'émergence de pratiques d'écriture pour soi qui ne se réfèrent pas à des genres établis de l'écrit personnel et qui émanent de scripteurs peu-lettrés. On y perçoit l'intrication de différentes strates de la culture écrite : écrits de l'encadrement agricole en bambara, documents officiels en français, culture lettrée islamique... Ces pratiques reflètent aussi la densité des circulations culturelles, entre écrit et oral, dans les sociétés même rurales du Mali contemporain. Si différents rapports à l'oral et à l'écrit sont décelables, ces

\footnotetext{
${ }^{2}$ Nous nous inspirons ici des distinctions conceptuelles mises en œuvre par Michel Foucault dans Histoire de la sexualité. 3, Le souci de soi, Paris, Gallimard, 1984, p. 59-60.

${ }^{3}$ Voir Sean HAWKINS, Writing and colonialism in northern Ghana: the encounter between the LoDagaa and "The world on paper", 1892-1991, Toronto, University of Toronto Press, 2002.

${ }^{4}$ Karin BARBER (dir.), Africa's hidden histories: everyday literacy and making the self, Bloomington, Indiana University Press, 2006, p. 7 ; K. BARBER, The anthropology of texts, persons and publics. Oral and written culture in Africa and beyond, Cambridge, Cambridge University Press, 2007.
} 
productions sont à prendre au sérieux comme d'emblée dans l'écrit, dans un rapport à des normes graphiques qu'il faut à chaque fois précisément identifier ${ }^{5}$.

Nous proposons d'aborder cette pratique par la question du support d'écriture. On connaît l'importance de cette thématique dans l'histoire culturelle et sociale du livre, dans la perspective ouverte par Donald McKenzie et développée par Roger Chartier ${ }^{6}$. L'étude du support fournit des outils essentiels s'agissant de l'écriture de cahiers tenus sur de longues durées par des scripteurs qui le maîtrisent inégalement. Même sur des terrains européens pour lesquels l'étude de la culture écrite constitue un domaine établi, Jean Hébrard constate qu'en tant que supports d'écrits personnels « carnets, cahiers, registres, journaux, albums (...) sont des objets qui sont en grande partie demeurés pour les chercheurs sans grand intérêt ${ }^{7} \gg$. Pour le domaine africain où les travaux sur les pratiques d'écriture sont émergents, on comprend que cette dimension n'ait jusqu'ici pas retenu l'attention des chercheurs ${ }^{8}$. L'objet de cet article est de montrer ce qu'une attention au cahier comme support apporte à une étude anthropologique de cette pratique.

La question du support amène à considérer le cahier d'abord comme un objet personnel que le scripteur détient. Puis, entrant dans cette boîte noire qu'est le cahier, l'attention sera portée à la manière dont le scripteur s'en empare : comment organise-t-il les données recueillies sur la succession de feuilles qui composent le cahier? Quelles sont les différentes manières d'écrire

\footnotetext{
${ }^{5}$ Sonia BRANCA-ROSOFF et Nathalie SCHNEIDER, L'écriture des citoyens : une analyse linguistique des peulettrés pendant la période révolutionnaire, Paris, Klincksieck, 1994 ; Jan BLOMMAERT, Grassroots literacy. Writing, identity and voice in Central Africa, London, Routledge, 2008.

${ }^{6}$ Donald F. McKenZIE, La bibliographie et la sociologie des textes, Paris, Editions du Cercle de la Librairie, 1991 ; Roger CHARTIER, Culture écrite et société. L'ordre des livres (XIV'-XVIII siècle), Paris, Albin Michel, 1996.

${ }^{7}$ Jean HEBRARD, «Tenir un journal. L'écriture personnelle et ses supports », in Récits de vie et médias, Philippe LEJEUNE (dir.), RITM. Recherches interdisciplinaires sur les textes modernes, ${ }^{\circ} 20,1999$, p. 9-50, ici p. 10. Voir tout de même, plus récemment, Philippe LEJEUNE et Catherine BOGAERT, Un journal à soi: histoire d'une pratique, Paris, Textuel, 2003.

${ }^{8}$ Cette remarque vaut pour les pratiques contemporaines sur papier. L'étude des manuscrits arabo-africains bénéficie de traditions de recherche mieux établies, même si Constant Hamès souligne que ce domaine est peu balisé, voir « Les manuscrits arabo-africains : des particularités ? », Revue des mondes musulmans et de la méditerranée, n99-100, 2002, p. 169-182.
} 
qui se constituent sous les contraintes particulières de ce type de support? En quoi éclairentelles les modes de subjectivation à l'œuvre à travers cette pratique ?

Un préalable indispensable est de présenter le contexte de cette enquête, en brossant les grands traits d'une situation où la maîtrise de l'écrit est inégalement partagée, et en décrivant la méthode retenue pour l'aborder.

\section{Enquêter sur des cahiers}

Les cahiers étudiés ont été recueillis au cours d'une enquête ethnographique sur les pratiques de l'écrit dans un village situé près de la ville de Fana 9 . Les pratiques de l'écrit sont définies comme l'ensemble des gestes de lecture, d'écriture et de manipulation d'écrits, imprimés ou manuscrits. L'enquête a été progressivement resserrée sur une pratique singulière, la tenue d'un cahier personnel.

Pour comprendre et situer cette pratique, il faut identifier les scripteurs de cahiers : s'agit-il d'une pratique partagée par l'ensemble des villageois alphabétisés ou est-elle spécifique à certains groupes ou à certaines périodes de la vie ?

\section{Etre alphabétisé au village}

Dans la région cotonnière, l'introduction massive de l'écriture remonte aux campagnes d'alphabétisation fonctionnelle des années 1970, liées à la réorganisation de la production cotonnière sous l'égide de la CMDT (Compagnie Malienne pour le Développement des Textiles).

L'incitation au développement de la culture du coton, traditionnellement pratiquée pour un marché artisanal local, remonte à la période coloniale et à la pression fiscale. Cependant,

\footnotetext{
${ }^{9}$ Cette étude s'inscrit dans une enquête plus large sur les pratiques de l'écrit dans la région qui a donné lieu à ma thèse de doctorat, Aïssatou MBODJ-POUYE, «Des cahiers au village. Socialisations à l'écrit et pratiques d'écriture dans la région cotonnière du sud du Mali », thèse de doctorat de Sociologie et Anthropologie de l’Université Lumière-Lyon 2, sous la direction de Bernard LAHIRE, soutenue le 16 mars 2007.
} 
contrairement aux expériences de culture irriguée du coton mises en place à partir de 1932 dans la région de l'Office du Niger par déplacements de population, la culture pluviale du coton dans la région méridionale demeure, durant cette période, faiblement encadrée ${ }^{10}$. L'après-guerre marque un tournant, avec des innovations techniques et la mise en place de l'encadrement agricole de cette zone ${ }^{11}$. La culture du coton pour l'exportation se développe à la fin de la période coloniale et s'accroît durant les années 1960 avec l'introduction par la CFDT de la culture attelée.

La CMDT, créée en 1974, s'engage dans un programme de développement rural plus vaste, qui repose sur le système des associations villageoises (AV) : au lieu de traiter avec chaque exploitant, la CMDT distribue en début de campagne semences et pesticides aux membres de $l^{\prime} \mathrm{AV}$, qui se chargent de répartir ces produits, pris à crédit, auprès des agriculteurs ${ }^{12}$. En fin de campagne, lors de la commercialisation du coton, le calcul des sommes à percevoir une fois acquittés les crédits s'effectue localement. Le cycle annuel d'une campagne est rythmé par des activités de mesure, de compte et d'écriture : mesure des superficies ; consignation des produits attribués à chaque exploitant et des crédits à rembourser ; pesée du coton, etc.

Comme la constitution d'un village en AV passe par la mise en place d'un bureau dont les principales fonctions (trésorier, secrétaire, magasinier) requièrent de manier l'écrit, la CMDT a organisé des sessions d'alphabétisation fonctionnelle en bambara dans les villages, puis des formations de post-alphabétisation sur des thèmes techniques ou d'organisation communautaire $^{13}$. Ces sessions ont touché un public surtout masculin, visant d'abord des

\footnotetext{
${ }^{10}$ Sur ces différentes expériences, voir ROBERTS Richard Two worlds of cotton. Colonialism and the regional economy in the French Soudan, 1800-1946 Stanford, Stanford University Press, 1996.

${ }^{11}$ Cette période est marquée par la création de l'IRCT (Institut de recherche du coton et des textiles exotiques) en 1946, puis de la CFDT (Compagnie Française pour le Développement des Textiles) en 1949.

${ }^{12}$ Voir SANOGO Bakary Le rôle des cultures commerciales dans l'évolution de la société sénoufo (sud du Mali) Bordeaux, CRET, 1989. Ce système, basé sur la fixation du prix de récolte en début de campagne, a fonctionné durant les années 1970 et 1980, années de formation des scripteurs étudiés, jusqu'à la crise du coton des années 1990 et la privatisation en cours de la CMDT.

${ }^{13}$ Le français est la seule langue officielle du Mali. Le statut de langue nationale est reconnu à plusieurs langues, parmi lesquels le bambara se distingue par son statut véhiculaire à l'échelle du pays. Voir Cécile CANUT, Dynamiques linguistiques au Mali, Paris, ACCT, Didier érudition, 1996. Le bambara utilisé pour
} 
agriculteurs susceptibles de prendre des responsabilités collectives ${ }^{14}$. Le système des AV et l'extension de la culture du coton pour la commercialisation ont eu des conséquences socioéconomiques extrêmement importantes, notamment sur le développement des champs individuels et l'accélération des processus de scission des exploitations familiales ${ }^{15}$.

Le village d'enquête se caractérise par une entrée précoce dans la dynamique de l'encadrement agricole et de l'alphabétisation ${ }^{16}$. Un centre d'alphabétisation fonctionnelle en bambara y a été ouvert dès 1971, sous l'égide de la DNAFLA (Division Nationale de l'Alphabétisation Fonctionnelle et de la Linguistique Appliquée) dans le cadre d'une campagne nationale d'alphabétisation en langues nationales à destination des paysans. L'alphabétisation y a été assurée par un des tout premiers scolarisés du village, né en 1928 et recruté pour l'école de Fana (en français) en 1937. En 1971, après une formation d'une semaine au bambara écrit, au chef-lieu de cercle, il est revenu au village doté de matériel didactique (manuels notamment) et chargé de rassembler les villageois volontaires pour l'alphabétisation en cours du soir. Ceux-ci ont suivi la forme très scolaire préconisée par les formateurs ${ }^{17}$. Cette période a été marquée par un fort investissement dans la postalphabétisation, avec notamment la diffusion de documents imprimés : calendriers, carnets

l'alphabétisation et la scolarisation est écrit en graphie latine, empruntant depuis la réforme de l'orthographe de 1982 quelques signes à l'alphabet adopté pour les langues africaines.

${ }^{14}$ Théoriquement ouvertes aux femmes, les sessions d'alphabétisation de la CMDT ont toujours réuni une majorité d'hommes. Des sessions d'alphabétisation féminine ont été mises en place plus tard par des ONG, avec un succès limité.

${ }^{15}$ Voir SANOGO Bakary Le rôle des cultures commerciales..., op. cit. et JONCKERS Danielle La société minyanka du Mali. Traditions communautaires et développement cotonnier Paris, L'Harmattan, 1987. Laurence BECKER, dans «The collapse of the family farm in West Africa? Evidence from Mali » The Geographical Journal 156, $\mathrm{n}^{\circ} 3,1990, \mathrm{p} .313-322$, discute l'idée selon laquelle la scission serait la seule réponse aux transformations liées au développement des cultures de rente, certaines grandes exploitations familiales se maintenant en accordant une plus grande autonomie économique aux individus qui les composent.

${ }^{16}$ Ce village, qui compte aujourd'hui près de 1500 habitants, a été fondé à la fin du XIXème siècle par des familles auparavant esclaves de Marka. Ce passé explique sans doute une ouverture vers des domaines où ce stigmate n'opère pas, contrairement à l'espace politique où la domination du village voisin, anciennement siège du canton (kafotigi), pèse encore fortement. Pour une discussion détaillée, voir Aïssatou MBODJ-POUYE, «Des cahiers au village »... op. cit., p. 153-163. Sur le caractère post-esclavagiste autant que postcolonial de la société malienne contemporaine, voir MANN Gregory Native sons. West African veterans and France in the twentieth century Durham, Duke University Press, 2006. L'échelle plus large à laquelle a été menée l'enquête pour ma thèse permet de caractériser ce village par la densité des pratiques d'écriture qui y sont présentes, mais ces mêmes pratiques, y compris la tenue d'un cahier à soi, sont attestées dans d'autres villages de la région.

${ }^{17}$ Voir la description de ces sessions près de Fana dans Bernard DuMONT, L'alphabétisation fonctionnelle au Mali : une formation pour le développement, Paris, Unesco, 1973. 
d'exploitation imprimés destinés à la consignation pour chaque exploitation agricole des principales informations agricoles ${ }^{18}$.

La dynamique d'alphabétisation a permis à la fois un passage rapide au système des $\mathrm{AV}$ promu par la CMDT, et le choix de ce village pour l'ouverture d'une école bilingue en 1979. Auparavant, la scolarisation avait touché le village de manière sporadique, à travers le recrutement de quelques enfants pour des écoles urbaines depuis la période coloniale. En 1974, une école ouverte dans le village voisin a marqué le début d'une scolarisation plus régulière, toujours en français. En 1979, dans le cadre d'une politique d'expérimentation des langues nationales à l'école, une école franco-bambara a été ouverte au village. L'école se distingue de l'alphabétisation par son recrutement élargi, touchant filles et garçons. Les trajectoires scolaires témoignent du seuil que représente la fin du primaire, avant la poursuite des études au second cycle à Fana : la plupart des scripteurs de cahiers ont un niveau qui se situe entre $6^{\text {ème }}$ et $7^{\text {ème } 19}$. Cette nouvelle génération de lettrés a partiellement pris la relève des premiers alphabétisés en bambara. Cependant l'école, fournissant des lettrés en excès sur les opportunités locales de valorisation des compétences, a aussi donné naissance à des profils de lettrés différents, qui exercent leur relative maîtrise de l'écrit dans des domaines privés, sans accéder par là à un statut particulièrement reconnu ${ }^{20}$.

Les années 1970 constituent donc un tournant avec une pression accrue à l'alphabétisation des adultes, la diffusion d'un ensemble de pratiques d'organisation du monde agricole où l'écrit est central, et le recours au bambara comme langue d'enseignement. Enquêter sur les

\footnotetext{
${ }^{18}$ Ibid. p. 10. Cette pratique n'a pas connu le succès espéré, et n'est pas attestée dans le village, mais l'existence de ce modèle témoigne de la volonté des premiers formateurs de constituer un environnement graphique.

${ }^{19}$ Le dépouillement des registres scolaires des trois premières cohortes montre qu'une majorité d'élèves (71 sur 125 ) n'atteignent pas la $6^{\text {ème }}$. Sur les 54 qui l'atteignent, 22 s'arrêtent en $6^{\text {ème }}$ (8 ayant eu l'examen) et 16 en $7^{\text {ème }}$, les parcours des autres étant contrastés (niveau $9^{\text {ème }}$ à études supérieures).

${ }^{20}$ Sur ce point, nous rejoignons les conclusions d'Etienne Gérard, qui montre que la maîtrise de l'écrit est devenue un enjeu, indépendamment de l'accès à l'emploi formel ou des opportunités sociales qu'ouvre la scolarisation. Voir Étienne GERARD, La tentation du savoir en Afrique. Politiques, mythes et stratégies d'éducation au Mali, Paris, Karthala-ORSTOM, 1997. En revanche la dynamique des langues et des systèmes éducatifs qu'il décrit (avec une préférence pour les médersas et un désintérêt pour les langues nationales) est différente dans la zone d'enquête, où le bambara écrit est valorisé par les usages promus par la CMDT.
} 
pratiques de l'écrit des adultes trente ans après ce moment de diffusion de l'écrit autorise à poser la question de la pérennité des usages et à étudier l'extension du recours à l'écrit à des domaines qui n'étaient pas initialement visés par l'alphabétisation. L'approche ethnographique de ces pratiques permet de contourner le débat sur l'évaluation quantitative des résultats de l'alphabétisation ou de la scolarisation ${ }^{21}$. Elle donne aussi l'occasion d'élargir la focale en appréhendant des pratiques qui puisent à des traditions lettrées et à des expériences de l'écrit variées.

En effet, la culture lettrée islamique est présente dans ce village, notamment par l'école coranique, premier stade de l'enseignement islamique traditionnel. Quelques années d'école coranique assurent une connaissance à l'oral de prières en arabe et des compétences graphiques réduites, parfois une aptitude à la copie. Pour quelques uns, l'apprentissage se prolonge hors du village. Pour les autres, la pratique de l'arabe se poursuit par la lecture de brochures islamiques qui associent à l'arabe (en graphie arabe ou en translittération latine) le bambara et le français ${ }^{22}$.

A ces différentes filières de formation s'ajoutent d'autres expériences de l'écrit, certaines plus anciennement présentes. L'administration repose sur des usages de l'écrit en français, qui s'appliquent à tous les individus, quelle que soit leur maîtrise effective de cette langue, à travers des procédures collectives (recensement) et individuelles (inscription à l'état civil ; délivrance d'un carnet de vaccination, etc.). Enfin, la migration saisonnière d'une grande

\footnotetext{
${ }^{21}$ Pour une évaluation des résultats de l'alphabétisation en zone CMDT, voir Klaudia DoMBROwSKY, Gérard DUMESTRE et Francis SIMONIS, L'alphabétisation fonctionnelle en bambara dans une dynamique de développement. Le cas de la zone cotonnière (Mali-Sud), Paris, ACTT, Didier Erudition, 1993.

${ }^{22}$ Sur ces brochures, voir Francesco ZAPPA, «Écrire l'Islam en bambara : lieux, réseaux et enjeux de l'entreprise d'al-Hâjj Modibo Diarra », Archives de sciences sociales des religions, à paraître en juillet 2009. L'ajami (écriture d'une langue africaine en graphie arabe) n'est pas pratiqué dans ce village. Sur la pratique de l'ajami dans l'aire mandé, voir cependant Valentin VYDRINE « Sur l'écriture mandingue et mandé en caractères arabes (mandinka, bambara, soussou, mogofin) » Mandenkan $\mathrm{n}^{\circ} 33,1998$, p. 1-87, et Tal TAMARI « Cinq textes bambara en caractères arabes : présentation, traduction, analyse du système graphique »Islam et sociétés au sud du Sahara, $n^{\circ} 8,1994$, p. $97-121$.
} 
partie des jeunes, garçons et filles, leur procure, en ville, des occasions de contact avec des écrits beaucoup plus divers ${ }^{23}$.

Chacune des formes d'alphabétisation en présence s'appuie sur des modes d'entrée dans l'écrit et instaure des rapports à l'autorité du texte singuliers : rapport étroit à l'oralité à travers récitation et apprentissage par cœur dans une langue au début inconnue, sacralisation du texte coranique dans l'enseignement islamique traditionnel ${ }^{24}$; entrée à la fois dans l'écrit et dans une langue, le français, pour la scolarisation, qui vise l'acquisition d'un savoir défini nationalement et l'obtention de titres censés ouvrir l'accès à l'emploi formel ; apprentissage revendiquant un ancrage local tout en empruntant à l'école l'essentiel de sa forme pour les sessions d'alphabétisation fonctionnelle en bambara. Pour autant, la pluralité d'expériences qui marque les trajectoires forge une culture écrite qui ne se réduit pas à la superposition de filières distinctes. Contrairement aux contextes où chaque langue est liée à une technologie (système graphique et dispositif matériel) et à des usages de l'écrit distincts ${ }^{25}$, ces formes d'alphabétisation sont étroitement imbriquées, et donnent lieu à des pratiques qui mêlent langues, graphies et genres issus de ces différentes cultures écrites.

\section{Constituer un corpus de cahiers}

L'enquête sur les pratiques de l'écrit a reposé sur différents outils.

La passation d'un questionnaire à l'échelle du village assure un aperçu global de la distribution de l'écrit selon les langues ${ }^{26}: 18 \%$ des adultes déclarent pouvoir écrire une lettre

\footnotetext{
${ }^{23}$ La zone connaît une faible émigration internationale si on la compare à d'autres régions du Mali: quelques villageois sont établis en Côte-d'Ivoire, en revanche on ne comptait en 2004 aucun émigré en Europe. Le phénomène migratoire saisonnier, à destination des villes ou des pays voisins, est quant à lui massif.

${ }^{24}$ Pris dans son ensemble et incluant les médersas, l'enseignement islamique offre une gamme beaucoup plus complexe de rapports à l'écrit. Voir Louis BRENNER, Controlling knowledge: religion, power, and schooling in a West African Muslim society, Indiana, Indiana University Press, 2001.

${ }^{25}$ Un cas exemplaire est celui des Vaï du Libéria étudiés par Sylvia Scribner et Michael Cole dans The Psychology of Literacy, Cambridge, Mass., Harvard University Press, 1981.

${ }^{26}$ J'ai passé ce questionnaire en me déplaçant dans chacune des 95 concessions du village. Les renseignements recueillis ont été fournis par la personne concernée si elle était présente, sinon par ses proches. Le critère retenu pour la mesure de l'alphabétisation (pouvoir lire ou écrire une lettre) tient compte de ce dispositif : faire lire ou
} 
en bambara, 9\% en français (pour l'essentiel également lettrés en bambara) et moins de 1\% (4 individus) en arabe $\mathrm{e}^{27}$.

Les entretiens semi-directifs $(n=56)$ ont d'abord été menés en suivant un réseau d'interconnaissance, à partir des responsables d'une des associations villageoises, qui ont signalé les personnes dont l'usage de l'écrit leur était connu. Dans ce premier temps, l'enquête a été orientée vers des individus aux compétences socialement reconnues : des hommes, chefs de famille, membres actifs de l'association villageoise, et les quelques femmes scolarisées dont les compétences sont sollicitées dès qu'une ONG cherche une interlocutrice alphabétisée. Dans un second temps, l'enquête a été menée auprès des anciens élèves des trois premières cohortes de l'école du village qui ont accompli un cycle primaire (entrés à l'école en 1979, 1981 ou 1983, le recrutement étant bisannuel) ${ }^{28}$. Ce second moment, systématique grâce au recours aux registres scolaires, a permis de rencontrer des villageois plus jeunes, dont les compétences, rarement sollicitées dans l'association villageoise, restent souvent inaperçues à l'échelle du village.

Les entretiens ont porté sur les parcours de formation et les pratiques actuelles dans les différentes langues ${ }^{29}$. Ils ont constitué des occasions d'observation des écrits, en particulier lors de scènes de lecture et de commentaire par les scripteurs de leurs cahiers professionnels et personnels. Ce dispositif de lecture commentée est une situation provoquée par l'enquête, qui ne correspond à aucun usage habituel du cahier ; cependant, ceux-ci ne sont pas écrits à ma demande. Tenus avant ma venue, ils ne sont pas liés à la relation ethnographique, sauf

écrire une lettre est un besoin courant, aussi sait-on généralement qui on peut solliciter pour une telle tâche. Sur les enjeux de la délégation d'écriture de lettres, voir Étienne GERARD, « Trajets d'écriture en Afrique. Le développement de la culture scolaire en questions », Communications, 72, 2002, p. 195-217. Pour un dispositif d'enquête statistique sur les compétences plurilingues sur un terrain voisin, voir Marie-Eve HUMERY, «Le paradis, le mariage et la terre : des langues de l'écrit en milieu fuutanke (arabe, français et pulaar) », Cahiers d'études africaines, XLI (3-4)-163-164, 2001, p. 565-594.

${ }^{27}$ Ces chiffres concordent avec le taux d'alphabétisation au Mali, estimé à 19\% en 2004 (PNUD).

${ }^{28}$ Sur les 54 anciens élèves concernés, 41 ont été vus en entretien, soit au village soit dans leur lieu de résidence actuel pour ceux qui sont installés à Fana ou Bamako.

${ }^{29}$ J'ai appris le bambara à l'INALCO pour ma thèse, mais n'ai pas de compétences linguistiques en arabe, ce qui explique que je n'ai pu enquêter précisément sur les usages de l'écrit dans cette langue. 
dans trois cas, où j'ai constitué des séries de cahiers au cours de différents séjours effectués au village entre 2002 et 2004 . Au cours des entretiens, la prise en photo totale ou partielle des cahiers présentés a été négociée, permettant de recueillir 424 pages photographiées.

\section{Une pratique partagée}

Les travaux sur les pratiques personnelles de l'écrit en Afrique reposent principalement sur des études de $\operatorname{cas}^{30}$. Notre recherche s'en distingue en portant sur une pratique relativement répandue parmi les villageois alphabétisés ${ }^{31}$.

Si la logique ethnographique du recueil interdit une exploitation statistique de ce corpus, le recours ponctuel à des données chiffrées donne tout de même une idée de l'extension de la pratique. Sur 43 enquêtés auprès desquels l'entretien a porté sur ces questions, 33 déclarent prendre des notes personnelles dans un cahier. Encore les déclarations ne permettent-elles pas toujours, nous y reviendrons, de saisir la pratique.

Dans ce village, cette pratique est transversale à l'ensemble des générations de lettrés : un des premiers villageois scolarisé, entré à l'école en 1942, puis recruté par la compagnie cotonnière comme moniteur d'alphabétisation, tient un ensemble de cahiers ; les plus jeunes enquêtés, d'une trentaine d'années, encore étudiants ou rentrés s'établir au village, comptent aussi parmi les scripteurs. Quant à leur formation, si la plupart des scripteurs de cahiers ont été scolarisés, d'autres sont passés uniquement par l'alphabétisation. Les profils associant école ou alphabétisation et formation islamique sont nombreux. Outre l'agriculture, activité principale de tous les villageois, deux autres domaines sont à signaler : le commerce, comme revenu d'appoint (déplacements à la foire hebdomadaire de Fana) ou régulier (tenue d'une

\footnotetext{
${ }^{30}$ Voir Karin BARBER (dir.), Africa's hidden histories..., op. cit. Une approche par cas est classique dans les travaux sur l'acculturation à l'écrit, comme le démontre l'étude de Carlo GINZBURG, Le fromage et les vers. L'univers d'un meunier au XVIème siècle, Paris, Flammarion, 1980.

${ }^{31}$ Une approche des écrits à l'échelle villageoise est fournie par le relevé de l'ensemble des écrits présents dans un village camerounais que propose David Zeitlyn dans «The documentary impulse: archives in the bush », History in Africa, 32-2005, p. 415-434. Sa discussion des pratiques s'appuie toutefois sur un dispositif différent puisqu'il fait tenir des cahiers par des villageois.
} 
échoppe au village); le travail dans l'association villageoise. Sans être des professionnels de l'écrit, certains scripteurs ont par là des usages quotidiens de l'écrit. Enfin, la pratique de tenir un cahier est attestée chez des hommes et des femmes, mais semble tout de même davantage sexuée que l'alphabétisation : sur les dix personnes qui ne tiennent pas de cahier, sept sont des femmes, pourtant retenues pour l'enquête sur les mêmes critères d'alphabétisation que les hommes.

Si la distribution sociale de cette pratique ne peut être établie de manière définitive, cet aperçu témoigne de la diversité des profils de scripteurs de cahiers, qui permet de porter sur cette pratique commune un regard attentif aux manières plurielles d'écrire. L'exploration du cahier comme support d'une écriture personnelle peut maintenant commencer, en considérant d'abord le cahier en tant qu'objet. Qu'est-ce qui distingue le cahier personnel des nombreux autres cahiers qui circulent au village ?

\section{Le cahier, un objet à soi}

\section{Les cahiers, principaux supports d'écriture}

Le cahier, utilisé tel quel ou comme un bloc dont on détache des feuilles, est un support d'écriture omniprésent au Mali, en milieu urbain comme rural. Objet manufacturé, le cahier est produit dans des imprimeries à Bamako ou dans d'autres grandes villes africaines, comme le montrent les couvertures en couleur où figure une carte du Mali ou la photo d'un joueur de football célèbre, et qui déclinent des tables de multiplication ou l'hymne national. Acheté en ville, il a un prix qui n'est pas négligeable pour des agriculteurs dont les revenus monétaires sont limités (200 F CFA pour un cahier de 96 pages).

Au village, au-delà de l'école et des sessions d'alphabétisation où il forme avec le tableau noir le support de l'apprentissage, ce cahier d'écolier (petit format, quadrillé à grands carreaux, de 48 ou 96 pages) est central dans les principaux domaines où l'écrit est présent. 
La CMDT recourt à une diversité de supports d'écriture, dont certains lui sont propres, comme les imprimés à remplir : bordereaux d'expédition, reçus, cahiers de caisse, etc. Mais la plupart des écrits quotidiens de l'association villageoise se font dans des cahiers d'écoliers. Ceux-ci sont consacrés à des usages spécifiques, que signalent ces titres, portés manuscritement sur la couverture : relevé de crédit («juru seben »), cahier de caisse («kesu kaye $»)^{32}$, etc. Certains sont transformés par des découpes manuelles pour prendre un format particulier.

L'administration associe elle aussi différents supports. Son travail repose sur des « chaînes d'écriture » qui aboutissent à des écrits officiels : actes et registres d'état civil conservés à la mairie située dans un village voisin ${ }^{33}$. Sur place, recensement villageois et consignation des naissances et décès figurent dans des cahiers tenus par le responsable administratif du village. Ces données de l'état civil apparaissent aussi, nous le verrons, dans les cahiers personnels.

Les différents intervenants extérieurs au village (CMDT et services administratifs, ONG) dispensent régulièrement des formations («kalan » en bambara) aux villageois alphabétisés. Les occasions et les thèmes les plus divers sont prétextes à formation : mise en place de l'association de parents d'élèves, tenue d'un bureau de vote, etc. La forme en est toujours très scolaire : un cahier est distribué en début de session, où les villageois convoqués copient ce que le formateur écrit au tableau. Les ONG et les banques paysannes qui mettent en place des programmes au village confient également des cahiers aux personnes à qui elles délèguent localement des tâches. Ces cahiers sont régulièrement inspectés lors des passages des responsables de l'organisation.

\footnotetext{
${ }^{32}$ La police utilisée pour le bambara a été développée par le LLACAN (Langage, langues et cultures d'Afrique noire - Inalco-CNRS).

${ }^{33}$ Nous reprenons la notion de chaîne d'écriture à Béatrice FRAENKEL, « Enquêter sur les écrits dans l'organisation », chap. 9, in Anni BORZEIX et Béatrice FRAENKEL (dir.), Langage et travail : communication, cognition, action, Paris, CNRS éditions, 2001, p. 231-261, ici p. 247-253.
} 
En dehors de ces domaines encadrés par des institutions, les supports de l'écriture personnelle ${ }^{34}$ déploient une variété de formats : calendriers suspendus au mur où l'on coche des dates, bouts de papier ou de cartouche de cigarettes récupérés pour y dresser des listes de courses, feuilles diverses, cahiers d'écoliers, carnets, bloc-notes offerts à titre de publicité, etc. Ce foisonnement est relatif, et les usages sont contraints par un souci d'économie de ces supports.

\section{Ecrire pour soi : feuille ou cahier, des usages différents}

On peut distinguer deux grands types de supports de l'écriture personnelle : les feuillets (feuilles de bloc ou de cahiers, papiers de format divers, bouts de cartons, etc.) et les supports reliés (cahiers, carnets, registres, bloc-notes, etc.), ces derniers pouvant être utilisés pour en détacher des feuilles.

A cette distinction correspond sans surprise une répartition des usages qui associe le feuillet à des écritures provisoires et réserve le cahier à des notations que l'on souhaite conserver. Prenons l'exemple des listes d'achats, qui se retrouvent sur des feuilles volantes et des pages de cahiers : les listes de courses qui accompagnent le déplacement à la foire de Fana, notamment pour consigner les commissions dont on est chargé, figurent sur des feuillets (feuilles de blocs ou bouts de cartons); dans les cahiers, ce sont des listes d'achats effectués qui apparaissent, quand le scripteur souhaite en garder trace.

Cette répartition globale connaît toutefois des exceptions. Certains usages du cahier sont mixtes, comme celui de la notation des crédits par Moussa Coulibaly : il consigne dans ses cahiers les crédits consentis en tant que tenancier d'une échoppe au village, qu'il barre au fur et à mesure de leur remboursement (opération qu'il effectue aussi sur des feuillets mobiles, qu'il jette une fois les crédits remboursés). Ce même cahier comporte par ailleurs des

\footnotetext{
${ }^{34}$ A ce stade, nous nous appuyons sur une définition par la négative de ces pratiques comme ne relevant pas des écritures imposées par les institutions. L'examen du contenu des cahiers, dans la partie suivante, permettra d'en proposer une définition plus précise.
} 
informations qu'il souhaite conserver sur une plus longue durée. Inversement, le cahier n'est pas toujours support exclusif de l'écriture personnelle, puisque certains scripteurs gardent précieusement des feuillets, dans leurs cahiers ou dans un livre. Dans l'ensemble, l'écriture pour soi a tout de même pour supports privilégiés cahiers et carnets.

\section{Réemplois}

Il peut sembler évident qu'écrire pour soi requière que le support soit d'emblée consacré à la notation personnelle. Pourtant, dans la plupart des cahiers qui composent notre corpus, la prise d'écriture ne coïncide pas avec l'ouverture d'un cahier. Le cahier est récupéré, souvent lors d'une formation. Parfois l'écriture est interstitielle, se logeant dans les marges et les espaces laissés vacants par une première strate d'écriture, par exemple dans un cahier scolaire. Cette manière de récupérer un cahier se prête à des écritures qui relèvent du brouillon ou ponctuellement de l'aide-mémoire. Le plus souvent, c'est d'une série de pages vierges que s'empare le scripteur. La copie lors des formations occupant rarement l'ensemble du cahier, l'espace laissé vacant après les notations est mis à profit pour un autre usage. La rupture liée au changement d'usage est quelquefois signalée graphiquement par le scripteur, par une page blanche ou un trait tiré.

Ce type de pratique est souvent apparu de manière détournée au cours des entretiens. Ainsi, Maïmouna Touré, une des femmes les plus lettrées du village, scolarisée jusqu'en $7^{\text {ème }}$, déclare dans un premier temps ne pas avoir de cahier personnel, avant qu'une description incidente de ses pratiques de notations personnelles ne l'amène à évoquer un cahier réutilisé.

MT C'est un cahier que j'ai comme ça, c'est moi-même qui ai... pour moi, j'ai pris un de mes anciens cahiers, j'écris comme ça (entretien traduit du bambara). 
Cette hésitation renvoie à une déclarabilité et une visibilité inégales des pratiques d'écriture ${ }^{35}$. Si les pratiques professionnelles et publiques, et dans le domaine privé des genres bien identifiés comme les lettres, sont aisément mentionnés, les écrits sur des supports mixtes sont difficiles à saisir.

\section{Un cahier « à part»}

Les cahiers d'emblée consacrés aux notations personnelles sont mieux identifiés. Bien qu'aucun nom générique ne soit donné au cahier à soi, les descriptions précisent qu'il s'agit d'un cahier « à part », pour reprendre une des expressions récurrentes.

Demba Coulibaly Bon, comme j'ai fait l'étude, et appris l'écriture là-dedans, donc tout ce que j'entends, si c'est important je vais l'écrire.

$A M$ D'accord. Et tous vos cahiers, c'est des choses comme ça ? (...)

$D C$ Non, non, non, j'ai... ça c'est personnel ça, pour le service y en a d'autres (entretien en français).

Demba Coulibaly, scolarisé jusqu'en CM1 selon le système alors en vigueur (il est né en 1932), a connu une carrière de moniteur au sein de la CMDT. Il a intériorisé une distinction entre professionnel et privé qu'il applique ici à ses écrits. D’autres enquêtés, sans avoir un travail caractérisé par des procédures d'objectivation aussi nettes (statut professionnel défini par poste et niveau d'études; temps de travail décompté; lieu séparé), partagent cette

\footnotetext{
${ }^{35}$ La question de la déclarabilité est classique concernant les lectures, voir Anne-Marie CHARTIER, Jocelyne DEBAYLE et Marie-Paule JACHIMOWICZ, « Lectures pratiquées et lectures déclarées : réflexions autour d'une enquête sur les lectures d'étudiants en IUFM », in Emmanuel FRAISSE (dir.), Les étudiants et la lecture, Paris, PUF, 1993, p. 73-98. Elle vaut aussi pour les pratiques d'écriture, pour lesquelles la hiérarchie scolaire des productions est intériorisée, qui disqualifie par exemple le brouillon. Au-delà de ce problème, celui de la simple visibilité est massif : comment saisir des gestes d'écriture inaperçus des scripteurs eux-mêmes? Voir Bernard LAHIRE, «Du travail d'enquête à l'écriture des paroles des enquêtés : réponse aux interrogations de Stéphane Beaud », Critiques sociales, n8-9-juin 1996, 1996, p. 108-114.
} 
habitude de répartir les cahiers selon les usages, qui va parfois jusqu'à une spécialisation des supports de l'écriture personnelle.

L'ouverture d'un cahier à soi peut être marquée matériellement sur le support. Les modifications artisanales de l'objet manufacturé, évoquées à propos des cahiers professionnels, prennent ici un tour singulier. Ainsi, l'épais cahier que Ba Madou Camara consacre à des notations religieuses est non seulement couvert de papier kraft, mais aussi renforcé par deux coutures (photo 1) : une bande de tissu soyeux soigneusement cousue sur le contre-plat renforce la pliure et un point de couture double une des agrafes centrales.

L'appropriation matérielle du support passe aussi par des dispositifs discursifs et graphiques qui ont pour site privilégié les espaces liminaires, reprenant l'usage scolaire de la page de garde, qui réserve la première page à l'identification du scripteur et du contenu. Paraphes et signatures qui émaillent les cahiers à soi sont récurrents sur le contre-plat ou sur la première page témoignant d'une personnalisation du support.

\section{Des histoires de cahiers?}

Les deux cas que nous avons distingués, réemploi ou ouverture d'un cahier, donnent-ils lieu à des usages suffisamment proches pour qu'on puisse les traiter comme une même pratique ? On pourrait supposer que le cahier ouvert pour soi donne lieu à des notations plus homogènes ou à une structuration plus maîtrisée de l'espace graphique ${ }^{36}$.

Concernant le contenu des cahiers, notre corpus fournit des exemples qui rapprochent les deux pratiques. Un cahier réutilisé peut donner lieu à une série de notations très homogènes, comme la série de formules magiques qu'Hinda Coulibaly consigne à la suite de pages copiées lors d'une formation. Deux autres cahiers, consacrés au même genre, sont eux,

\footnotetext{
${ }^{36}$ Cette notion est empruntée à Jean HeBRARD, « The Graphic Space of the School Exercise Books in France in the $19^{\text {th }}-20^{\text {th }}$ century », in Clotilde PONTECORVO (dir.), Writing Development. An interdisciplinary view, Amsterdam, John Benjamins, 1997, p. 173-189.
} 
ouverts par les scripteurs pour leur propre usage. Inversement, la notation fragmentaire est attestée dans les deux types de cahiers.

Quant à l'appropriation d'un espace graphique, le cahier ouvert pour soi offre certes des possibilités plus grandes, et la constitution d'un ordre lié à la succession temporelle des événements, examinée en détail ci-après, semble favorisée par le fait de consacrer un cahier à l'écrit pour soi, même si des chroniques ramassées se logent aussi dans des cahiers récupérés. Un point s'esquisse à partir des deux séries de cahiers conservées dans la durée par des scripteurs que j'ai pu observer ${ }^{37}$ : dans les deux cas, l'acquisition d'un cahier dédié aux notations personnelles intervient postérieurement à une prise d'écriture entamée sur d'autres supports. L'hypothèse d'une « carrière de scripteur », où le passage au cahier consacré à la notation personnelle marquerait une étape, demande à être approfondie à partir de données plus fournies, qui permettraient de poser la question de l'entrée dans l'écriture personnelle et de son évolution ${ }^{38}$.

\section{Le cahier, un objet à soi}

Récupéré ou non, le cahier est caractérisé, au moment de l'écriture personnelle, par le fait qu'il est la propriété du scripteur. Notre première caractérisation du cahier «à soi » repose sur le support, pris non pas dans sa seule matérialité (dans ce cas on retiendrait tous les cahiers, quels qu'ils soient), mais en tant que le scripteur s'en saisit pour ses propres besoins, soit par son acquisition, soit par un geste d'appropriation, au sens littéral, de cet objet. En ce sens,

\footnotetext{
${ }^{37}$ La rareté de ces séries tient au fait qu'archiver ses cahiers n'est pas la règle. Voir Aïssatou MBODJ-POUYE, «Pages choisies. Ethnographie du cahier d'un agriculteur malien », Sociologie et sociétés, vol. XL, n², automne 2008 : 96-108.

${ }^{38}$ Cette hypothèse rejoint la description qu'Elizabeth Gunner donne de l'entrée en écriture personnelle d'un membre d'une congrégation sud-africaine : d'abord support d'une pratique routinière de copie d'hymnes, le cahier devient lieu d'expression des visions du scripteur confronté à une crise spirituelle sans précédent, voir « Keeping a Diary of Visions: Lazarus Phelalasekhaya Maphumulo and the Edendale Congregation of AmaNazaretha », in Karin BARBER (dir.), Africa's Hidden Histories ...op. cit., p. 155-179. A la différence des autres études de journaux personnels réunies dans cet ouvrage, la contribution d'E. Gunner pose un problème comparable à notre cas puisqu'il s'agit de comprendre « comment quelqu'un se met à tenir un journal quand personne n'en a jamais suggéré la possibilité ?», ibid., p. 155 (notre traduction).
} 
«tenir » un cahier c'est d'abord le détenir. Un scripteur désigne ainsi son cahier personnel simplement comme «lasagolen», conservé ${ }^{39}$. Le cahier à soi se distingue des autres en ce que le scripteur le range dans ses affaires, dans l'espace personnel qui lui revient et qui se réduit souvent à une sacoche suspendue à un clou, une malle ou un coin sous un lit ${ }^{40}$. Objet mobile, le cahier suit certains scripteurs dans leurs déplacements (aux champs pour prendre note des superficies plantées, en voyage pour noter adresses utiles et consigner gains journaliers ...). D’autres, pour qui le souci de le préserver prime sur la nécessité d'en disposer régulièrement, le conservent à demeure chez eux.

Pratiquement toujours autographe, le cahier est le lieu d'une écriture pour soi ${ }^{41}$. Le scripteur peut y avoir recours ponctuellement lors d'une discussion, mais non en offrir une lecture continue $^{42}$. Un seul scripteur m'a indiqué avoir prêté le sien à un ami. Si le cahier est d'abord écrit pour soi, ces usages du cahier ne résolvent pas entièrement la question de l'adresse, et n'annulent pas les enjeux de présentation de soi, qui restent cruciaux dans l'écriture personnelle ${ }^{43}$. Comment comprendre l'accès relativement aisé que j'y ai eu ? Certes, quelques cahiers m'ont été cités mais non montrés, identifiés sans surprise comme «secrets»désignation qui permet soit de mettre un terme à la demande, soit au contraire de faire sentir son prix. Les rares réserves concernant la prise en photo ont porté sur des notations concernant des événements à venir, ou renvoyant à des situations délicates (fugue d'une

\footnotetext{
${ }^{39}$ L'anglais « to keep a diary » rend bien cette association première entre la possession du support et le type d'écriture qu'elle rend possible sur la durée.

${ }^{40}$ Je n'ai pas approfondi la question de la place des écrits au sein de l'espace domestique, dont Valérie Feschet a montré tout l'intérêt, voir «Textes et contextes. Les lieux de la mémoire dans les maisons ubayennes », Sociétés \& Représentations, $\mathrm{n}^{\circ} 19,2005$, p. 15-32. La conservation des écrits dans un espace intime restreint est le point que Karin Barber retient dans sa qualification du domaine de l'écrit personnel comme « tin-trunk literacy », en référence aux malles en fer-blanc qui contiennent les écrits à soi, voir son introduction à Africa's Hidden Histories...op. cit., p. 2.

${ }^{41}$ Quelques cahiers comportent ponctuellement des notations écrites par d'autres, mais la délégation d'écriture ne donne pas lieu à la tenue d'un cahier, contrairement à ce qu'Armando Petrucci a montré pour le livret de Maddalena Grattaroli, tenu pour sa charcuterie à Rome au XVI $\mathrm{X}^{\mathrm{e}}$ siècle, où il repère les mains de 102 scripteurs Armando PETRUCCI, «Scrittura, alfabetismo ed educazione grafica nella Roma del primo Cinquecento. Da un libretto di conti di Maddalena pizzicarola in Trastevere », Scrittura e Civiltà, 2, 1978, p. 163-207.

${ }^{42}$ On est loin de la performance orale du cahier devant un public, certes limité au cercle familial, décrite par Stephan MiESCHER, «"My Own Life": A. K. Boakye Yiadom's Autobiography. The Writing and Subjectivity of a Ghanaian Teacher-Catechist », in Karin BARBER (dir.), Africa's Hidden Histories...op. cit., p. 27-51.

${ }^{43}$ Voir Philippe LeJEUne, Le pacte autobiographique, Paris, Le Seuil, 1975.
} 
épouse ; coût des soins d'une maladie). Montrer son cahier, voire le laisser photographier, par une étrangère, extérieure au village, semble au premier abord étrange, gênant à considérer les fautes que je ne manquerai pas d'y repérer, mais parfois aussi valorisant. Quand bien même il s'agit de secrets, cela reste sans conséquence. Plusieurs enquêtés ont justifié le fait de me montrer certains de leurs secrets (notamment des « kilisiw », incantations magiques) par la certitude que je ne pourrais rien en faire, faute d'en comprendre les modalités d'usage :

$A M \mathrm{Si}$, ça ne vous dérange pas, est-ce qu'il y a des choses qu'on peut regarder?

$D C$ Oui, ça ne me dérange pas, parce que celui qui travaille, utilise ces choses-là c'est lui le propriétaire, marquer comme ça dans le cahier sans utiliser ça n'aboutit rien, n'est-ce pas ? Je peux vous montrer... (entretien en français).

\section{Du public au privé}

Les cahiers personnels échappent aux formes d'inspection et de contrôle auxquelles sont soumis les autres cahiers et registres villageois. Pour autant, leur contenu ne les distingue pas toujours nettement de ceux-ci : recension de naissances et décès ; comptes domestiques ; partage de la récolte familiale de coton; dates de semis... Ces notations, en français ou en bambara, constituent une part importante des écrits dans les cahiers à soi sans faire ressortir la spécificité de cette forme d'écriture.

On repère certes un changement d'échelle, consacrant le transfert de pratiques collectives, destinées à l'association de producteurs de coton ou à la communauté villageoise, à un espace privé, dont la principale dimension est la famille. Envisager ce déplacement comme un processus d'appropriation permet d'attirer l'attention sur le geste par lequel le scripteur se ressaisit de formes communes pour son usage, rappelant que l'écrit personnel n'est pas à rechercher au plus loin des injonctions à écrire mais dans la manière dont le scripteur 
s'empare de modèles qui lui sont imposés ${ }^{44}$. Au repérage des modèles scripturaux, genres et types de textes, formats d'écriture et normes graphiques, l'enquête doit alors associer la qualification du rapport à ces modèles pour appréhender la dynamique de l'appropriation ${ }^{45}$. Cette notion vise à saisir, au-delà de la dimension personnelle de la pratique, la façon dont le scripteur met en place et expérimente de nouveaux rapports à soi et aux autres, aux institutions et aux savoirs.

Trois cas nous permettront d'appréhender la manière dont ce passage du public au privé s'opère sur le support du cahier. On questionnera d'abord la notion de modèle, centrale dans une analyse en termes d'appropriation. Les échelles que recouvrent ou donnent à voir les notations du cahier seront ensuite scrutées, afin d'identifier le rôle constituant que cette pratique peut prendre. Enfin, à travers un cas de copie, on envisagera le processus d'appropriation dans son environnement graphique immédiat et dans la succession des gestes qui le composent.

\section{S'approprier un modèle : un « contrat » sur un cahier}

Nous avons vu Demba Coulibaly, dans l'extrait d'entretien cité plus haut, identifier précisément certains de ces cahiers comme «personnels ». Parmi ceux-ci, son cahier en cours, acheté pour ses propres besoins, comporte des notations variées : des prières en arabe, copiées d'un ouvrage qu'il détient, alternent avec des formules magico-médicinales, une adresse, et des notes concernant son exploitation agricole, comme celle-ci (photo 2$)^{46}$.

\footnotetext{
${ }^{44}$ Nous nous inspirons des travaux de Roger Chartier, en particulier Culture écrite et société ... op. cit. 213-215.

${ }^{45}$ Florence Weber résume ainsi les acquis des travaux français d'histoire et de sociologie de l'écrit : «les frontières ne sont ni entre écrit et oral, ni entre écrit ritualisé et écrit personnel, mais bien plutôt entre une plus ou moins grande distance avec les modèles proposés par l'institution, sans trancher pour l'instant la question de savoir si cette distance est subie (ignorance des modèles) ou volontaire (refus de modèles connus), si la proximité est familiarité, aisance (...) ou soumission » Florence WEBER, « La lettre et les lettres : codes graphiques, compétences sociales. Des outils pour l'analyse des écritures ordinaires », Genèses, 18, 1995, p. 152-165, ici p. 157.

${ }^{46} \mathrm{La}$ transcription respecte l'orthographe originale.
} 
- Début du travail de A.* K.*47

Le 8/ Mai/ 2002 (10 000 frcs par moi)

- araba domba mankon tile 24 don

san 2002 (10 000 francs par moi)

Fin 8 Aout 2002

$$
\text { D. }{ }^{*} \text { Cly }
$$

[signature manuscrite]

Ce type de notation est courant. Il transcrit les principaux éléments d'une transaction banale autour d'une prestation de service : un propriétaire de bétail confie son troupeau à un pasteur pour qu'il le mène sur des terres éloignées quand les pâturages se raréfient autour du village. L'utilité de la notation est évidente : le retour du pasteur intervient souvent après plusieurs mois, et le calcul du paiement suppose un décompte du temps travaillé et un rappel du salaire convenu. Ces notes sont souvent laconiques, réduites à des aide-mémoire personnels. Celle-ci se singularise par un ensemble de traits stylistiques, linguistiques et graphiques.

La formulation évoque par son souci de précision des écritures administratives ou juridiques : elle est explicite («Début du travail... Fin...»), donne la date de début et précise la somme convenue. La date de fin a été visiblement ajoutée lors du retour du pasteur, et clôt l'affaire. Le texte est pour l'essentiel en français. Une signature, précédée de la mention du nom, est apposée au-dessous ${ }^{48}$.

La date de début est notée dans deux calendriers : le calendrier grégorien, calendrier civil en vigueur au Mali, et le calendrier lunaire localement utilisé ${ }^{49}$. Mettre ces deux calendriers en

\footnotetext{
${ }^{47} \mathrm{~L}$ 'astérisque signale les abréviations qui sont de notre fait, afin de respecter l'anonymisation des individus. La plupart des patronymes étant très communs au Mali, $\mathrm{j}$ 'ai limité l'anonymisation aux prénoms des scripteurs.

${ }^{48}$ Notons qu'aucune description du bétail (nombre de têtes confié par exemple) n'apparaît. On sait que la question du décompte des têtes de bétail possédé, qui entre dans le calcul de l'impôt, est sensible.

${ }^{49} \mathrm{Ce}$ calendrier lunaire (dont les noms de mois bambara sont désignés génériquement par l'expression « farafin kalo », mois africain), antérieurement calé sur les saisons, coïncide aujourd'hui avec le calendrier musulman. La
} 
correspondance est une pratique récurrente dans les cahiers, en particulier pour les dates de naissance. La mise en correspondance est destinée à éviter toute contestation liée à une confusion de dates, le calendrier lunaire étant utilisé dans les échanges oraux qui président à la transaction ${ }^{50}$. La date de fin ne donne d'ailleurs pas lieu à la transposition dans ce calendrier.

La transposition se double d'une traduction : la date est donnée en français dans le calendrier grégorien, en bambara dans le calendrier lunaire. Le bambara se distingue graphiquement par son écriture en script et tout en minuscule, selon la norme graphique des premières formations d'alphabétisation. Le recours au bambara dans ce texte en français est circonscrit à cette mention.

L'ensemble fait l'objet d'une structuration graphique forte: mise en forme par deux tirets distinguant les deux versions de la date ; parenthèses ; signature qui se détache après un saut de ligne (ligne initialement laissée disponible, sans doute en prévision de l'ajout ultérieur de la date de fin). L'usage des tirets pour séparer quantième, mois et année, alors que la mention du mois en toutes lettres inciterait à une écriture continue produit un effet d'hypercorrection graphique.

Le style et la forme graphique pointent donc vers le modèle du contrat. Ce type d'écrit, sous forme imprimée ou manuscrite, fait partie des documents détenus par des chefs de famille au village (contrat de vente d'une bicyclette par exemple). Pourtant, plusieurs indices suggèrent que la visée n'est pas de produire un document au statut juridique achevé : l'abréviation, en particulier celle du nom propre du signataire, même si elle reprend une forme commune pour

mise en correspondance date, selon les régions du $\mathrm{XIX}^{\mathrm{e}}$ ou du début du $\mathrm{XX}^{\mathrm{e}}$ siècle selon Maurice DeLAFOSSE, «L'année agricole et le calendrier soudanais », L'Anthropologie, tome 31, 1921, p. 105-113. Sur la fluidité des usages actuels, entre calendrier agricole et calendrier musulman, voir Monique CHASTANET, « Entre bonnes et mauvaises années au Sahel. Climat et météorologie populaire en pays soninké (Mauritanie, Sénégal) aux XIXe et XXe siècles », in E. Katz, A. Lammel et M. Goloubinoff (dir.), Entre ciel et terre. Climat et sociétés, Paris, IRDEditions, 2002, p. 189-209. Pour une discussion plus large, notamment de la question de la concurrence entre calendriers lunaires ou basés sur le cycle des saisons, voir «Calendriers d'Afrique », Systèmes de pensée en Afrique noire, cahier 7, Paris, EPHE, 1984.

${ }^{50}$ On attendrait aussi conversion de la somme des francs CFA au doromè (=5 F CFA), unité de compte généralement utilisée à l'oral, et que Demba emploie pour une autre notation. 
ce nom; l'absence de validation du document par le pasteur (qui même non lettré pourrait apposer son empreinte) ; la rédaction du texte sur un cahier personnel et non sur un feuillet, éventuellement dupliqué. Ces écarts au modèle ne sont pas de l'ordre du détournement. Loin de la dérision ou de la contrefaçon, la note de Demba vise à reproduire pour son propre compte la rigueur de l'écrit officiel.

La validation inachevée par la signature, précédée d'une mention abrégée du nom, reste tout de même énigmatique, et conduit à s'interroger sur la validité de ce document en cas de contestation. Plusieurs enquêtés évoquent le recours à leur cahier comme modalité possible d'attestation de l'exactitude d'une date ou d'un montant lors de discussion internes à la famille ou au village, sans que j'aie pu déterminer si la production d'un tel document suffit à clore une discussion ou à l'emporter face à d'autres témoignages. Le recours à la justice est extrêmement rare et aucun cas où ce type d'écrit ait fait preuve ne m'a été cité ${ }^{51}$.

La signature ne sert sans doute pas tant à valider le document au regard d'une instance extérieure qu'à investir l'écrit personnel d'un peu de l'autorité de l'écrit officiel. Celle-ci est mise au service d'une présentation soignée, à travers laquelle Demba démontre sa maîtrise de ce type d'écrit (la signature est une aptitude distinctive) et de l'écriture en français. Le passage au bambara et au calendrier lunaire se fait en direction du pasteur, le français étant, ici comme ailleurs dans le cahier, la principale langue d'énonciation ${ }^{52}$. Le fait que la clôture de la transaction soit signalée par l'ajout de la date de fin (en français seulement) indique l'enjeu pour le scripteur à conserver sur son cahier cette trace dans son intégrité (par opposition à l'habitude de raturer des crédits remboursés ou des affaires closes).

\footnotetext{
${ }^{51}$ La question de son usage juridique reste à explorer, les conflits entre propriétaires et pasteurs, récurrents, étant parfois portés devant les tribunaux. Richards Roberts note que la rédaction de contrats écrits était encouragée par l'administration coloniale, ce qui suggère qu'une injonction à écrire a pu exister Richards L. ROBERTS, Litigants and households: African disputes and colonial courts in the French Soudan, 1895-1912, Portsmouth, Heinemann, 2005, p. 193.

${ }^{52}$ Pour les scripteurs qui écrivent dans les deux langues, le français est généralement préféré au bambara pour les écrits les plus personnels. Ce résultat, qui s'appuie sur la mise au point d'outils de description de l'alternance de langue spécifiques à l'écrit, a été établi en collaboration avec Cécile Van den Avenne. Voir Aïssatou MBODJPOUYE et Cécile VAN DEN AVENNE, « "C'est bambara et français mélangés". Analyser des écrits plurilingues à partir du cas de cahiers villageois recueillis au Mali », Langage et société, n¹20-juin 2007, 2007, p. 99-127.
} 
Cet exemple constitue un cas limite dans notre corpus par son rapport ambigu au modèle, entre reprise et imitation. Mettant en œuvre la pratique, récurrente, de l'emprunt d'un modèle, il permet de souligner le jeu qu'introduit l'écrit pour soi dans une culture écrite tramée de relations d'imposition, le scripteur investissant pour son propre compte l'autorité de l'écrit à laquelle il est d'ordinaire soumis.

\section{Circonscrire des espaces et des temporalités : l'espace graphique du cahier}

L'échelle de la notation ou de la page est insuffisante pour comprendre dans sa spécificité la tenue d'un cahier. L'observation de l'appropriation matérielle du support a déjà fait ressortir l'importance des espaces liminaires dans la prise du cahier et des points de transition dans les cas de réemploi. La succession des pages constitue le cahier en un espace graphique dont les scripteurs se saisissent diversement.

Les cahiers sont souvent tenus sur plusieurs années et font référence à des événements qui couvrent de longues périodes. Ces deux dimensions temporelles, temps de l'écriture, temps des événements, s'articulent diversement selon les cahiers, mais jamais sur le mode de la chronique à dates fixe : dans tous les cas, ce sont les événements qui dictent l'écriture, qui de ce fait est souvent sporadique ${ }^{53}$. A ces deux dimensions, il faut ajouter celle qui suit la linéarité du support, la troisième dimension du codex ${ }^{54}$.

L'ordre linéaire de lecture ou d'écriture du cahier ${ }^{55}$ constitue un axe temporel que le scripteur doit prendre en compte, soit qu'il en use comme d'un ordre chronologique implicite, soit qu'il lui donne une autre signification, comme le fait Makan Camara dans son cahier personnel ${ }^{56}$.

\footnotetext{
${ }^{53}$ Sur ce point, les cahiers diffèrent des agendas paysans étudiés par Nathalie Joly. Voir notamment « Ecrire l'événement : le travail agricole mis en mémoire » Sociologie du travail 46 (4), p. 511-527.

${ }^{54}$ Jean HÉBRARD, « Tenir un journal... », art. cit., p. 12.

${ }^{55}$ Il s'agit ici des cahiers en français et en bambara. Le choix d'un ordre d'écriture pour les cahiers qui associent graphies arabe et latine s'avère plus complexe.

${ }^{56}$ Nous avons proposé ailleurs une analyse approfondie de ce cahier, accompagnée de sa transcription intégrale, voir Aïssatou MBODJ-PouYe, « Pages choisies... », art. cit.
} 
Celui-ci s'ouvre sur des notations liées aux funérailles de son père en 1996. Trois autres pages rendent compte d'une répartition claire entre événements familiaux (p. 3: naissances et mariage entre 1996 et 2001, photo 3), événements nationaux ou internationaux (p. 4 : décès de Pépé Kallé, chanteur congolais, entendue à la radio ; mort d'un marabout célèbre au Mali, également consignée suite à l'écoute de la radio en 1998), faits marquants de la vie du village (p. 5 : inauguration d'une mosquée; décès du chef de village, en janvier et juillet 1998). Une dernière page comporte des notations plus hétérogènes (p. 6 : achats personnels en 1998 ; décès d'une personne non identifiée en 2000).

Si l'on observe attentivement les dates des faits consignés on repère premièrement que l'ordre chronologique du premier événement de chaque page correspond à l'ordre du cahier. Makan n'a pas laissé de pages vacantes, mais a « ouvert » les pages les unes après les autres, comme autant d'espaces d'écriture, en suivant la linéarité du cahier, et en respectant l'ordre chronologique à l'intérieur de chaque page (de l'événement le plus ancien en haut au plus récent en bas). Deuxièmement, les quatre pages qui se réfèrent à plusieurs événements (p. 3 à 6) portent sur des séries de faits dont les temporalités se chevauchent : Makan a écrit dans le même temps sur les différentes pages ouvertes, et y a classé les événements selon un ordre qui lui est propre.

Apparaît alors un mode de classement des événements rigoureux, qui donne consistance à des espaces sociaux relativement attendus: la famille, le village et au-delà ${ }^{57}$. Le travail de l'écriture dans la durée est toutefois loin d'être toujours aussi visible à l'échelle du cahier, et se laisse parfois mieux approcher à travers les pratiques qui mettent en rapport les différents supports de l'écriture personnelle, notamment l'activité de copie qui nourrit une large part des cahiers.

\footnotetext{
${ }^{57}$ A y regarder de plus près, l'échelle domestique retenue n'est toutefois la reproduction d'aucune des deux dimensions reconnues par la CMDT(l'exploitation) ou l'administration (la famille reconnue par le carnet de famille). Makan Camara recense en effet les naissances de ces épouses de manière exhaustive, mais aussi certaines des celles de ses belles-sœurs, résidant dans la même concession $(d u)$, mais relevant de l'exploitation de son frère aîné.
} 


\section{Noter un décès : copie et mise en mémoire}

Moussa Coulibaly se caractérise par l'intensité de ses pratiques de copie et la pluralité des supports dont il use en même temps. Agé de 30 ans au moment de l'enquête et scolarisé à l'école bilingue jusqu'en $6^{\text {ème }}$, Moussa Coulibaly est un villageois avec lequel je me suis liée d'amitié. J'ai photographié un grand nombre de ses écrits : trois cahiers, trois carnets, et des dizaines de feuillets (surtout des listes de courses). Il s'agit d'un des cas où la relation ethnographique a eu un effet sur la pratique. En particulier, son deuxième carnet, intitulé « carnet de secrets », est un carnet que j'avais emporté lors de mon premier terrain, et que je lui avais donné en cadeau en partant, n'en ayant pas eu besoin et ayant constaté qu'il faisait usage de ce type de support. Ce cadeau, fait sans arrière-pensée ni consigne d'écriture de ma part, témoigne de l'influence que notre relation a eue sur sa pratique, sans la susciter toutefois puisque Moussa Coulibaly écrivait déjà régulièrement avant notre rencontre. Ce carnet, que j'ai lu avec lui lors de mes séjours ultérieurs ne m'est pas destiné, et comporte des passages que Moussa Coulibaly n'a pas souhaité m'expliquer ainsi que des crédits et adresses visiblement à usage personnel. S'il a sans doute anticipé cette lecture possible, elle n'a pas à elle seule guidé l'écriture.

Dans ses différents cahiers, si l'on excepte les pages entières couvertes de crédits, ses notations personnelles sont pour l'essentiel des copies. Ses sources imprimées sont diverses : une grammaire française; des prières tirées d'une brochure franco-arabe; des titres de chansons sur une page qui reprend des pochettes de cassettes, les listes étant titrées «face $A$ », «face $\mathrm{B} »^{58}$; son extrait d'état civil qu'il recopie sur deux pages d'un de ses carnets, imitant la signature de l'officier d'état civil. Ses écrits sont également caractérisés par des pratiques de copie d'un cahier ou carnet à l'autre, invitant à considérer comme espace graphique, au-

\footnotetext{
${ }^{58}$ La notation de titres de chansons donne à lieu à toute une série de pratiques : noter des titres de chansons entendues à la radio pour se faire établir une compilation pirate, pour envoyer des dédicaces à la radio, etc.
} 
delà du cahier, l'ensemble de ses supports personnels. Quatre textes figurent en plusieurs versions : les résultats de la Coupe de monde de football sur deux double pages d'un de ses cahiers ; deux incantations en double et triple exemplaire ; la consignation de la mort de sa belle-sœur, décédée de suites de couches, dans deux de ses carnets ${ }^{59}$.

Nous allons nous arrêter sur ce dernier écrit (photos 4 et 5).

$1^{\text {ère }}$ version :

Mercredi Le 16 Octobre 2002-10

L'Acouchement de Worokiya

A Fana

Elle est decede Mardi le

29 Octobre.

A Fana

$2^{\text {ème }}$ version :

Mercredi 10. Le 16 Octobre 2002

L'Acouchement de Worokiya

est fait dans le dispensaire

de Fana vert 6 heur du mattain.

Elle est auppérrée

Worokiya est dessede

Le 29 Octobre 10 mois Mardi

Vert 8 heur A Fana

\footnotetext{
${ }^{59}$ Sur la copie, voir Christine BARRÉ-DE MINIAC (dir.), Copie et modèle : usages, transmission, appropriation de l'écrit, Paris, INRP, 2000.
} 
Ces deux textes ne constituent qu'une partie de la chaîne d'écriture à laquelle cet événement a donné lieu : Moussa Coulibaly a d'abord porté des encoches au bic sur un calendrier face aux deux dates de l'accouchement de Rokia et de son décès, avant d'en noter le détail dans un premier carnet, puis dans le carnet de secrets. Ces pratiques d'écriture en plusieurs temps, avec pour point de départ des prises de notes provisoires, reportées ensuite sur un support plus pérenne, sont souvent décrites par les scripteurs. Elles inscrivent l'écriture du cahier dans un environnement graphique dont la revue des supports proposée plus haut donne un aperçu.

Les deux versions du décès de Rokia diffèrent. Des détails sont précisés dans la seconde, qui ne sont pas indiqués pas dans la première (heure et lieu de l'accouchement, heure du décès) ; la seconde est plus soignée, dans la rédaction (énoncé complet et non tournure nominale) et dans l'écriture manuscrite, plus sobre (pas d'encre rouge); un fait central apparaît: l'opération. La première version peut avoir été écrite au fil des deux événements (le changement d'encre suggère des temps d'écriture distincts), la seconde leur est postérieure. Elle constitue un des rares textes de notre corpus à propos duquel on peut parler d'un récit : il a un début et une fin ; on y repère un travail d'écriture, la mention de l'opération dramatisant le propos. L'expression du décès reprend le prénom (le prénom seul et nom l'identité complète qui apparaîtrait sur un avis de décès), dans une formulation de l'ordre du constat clinique : «Rokia est décédée ».

Moussa Coulibaly conserve soigneusement ses différents carnets : la dernière version n'annule pas la première, et la copie ne peut s'expliquer simplement par le souci de garder trace de l'événement. La précision dans les détails et la forme graphique plus achevée attestent d'un travail d'écriture qui s'appuie sur le recours à d'autres sources (orales ou écrites, tel le certificat de décès). Le texte final témoigne d'une application dans la rédaction et l'écriture manuscrite. Cette dernière version apparait au début du «carnet de secrets » présenté plus haut, que Moussa Coulibaly emporte avec lui dans ses migrations saisonnières 
en ville. Ce carnet a progressivement été consacré à la notation de crédits ou d'informations diverses lors de ses déplacements hors du village. Mais il comporte aussi des notations qui composent une sélection d'écrits importants à conserver avec un soin tout particulier ou à avoir toujours sur soi : copie de sa carte d'identité, adresses, copie de formules magiques, et ce texte. Une fois déployé le travail d'écriture qui l'a produit, travail qui s'appuie sur plusieurs sources et supports et trouve son aboutissement sur un support intime, cet écrit apparaît aussi comme le fruit singulier d'un travail de mémoire.

\section{Un espace personnel}

Au terme de l'exploration de ces cas, deux tensions constitutives de la pratique de tenir un cahier apparaissent.

Premièrement, du public au privé, la position du détenteur du cahier change : celui-ci n'est plus seulement soumis à des inscriptions et à des écrits du contrôle, il dispose en tant que scripteur d'une relative liberté dans la sélection des notations, leur organisation dans le cahier et leur mise en forme. Certes, reprenant des modèles le plus souvent institutionnels, il se situe dans la continuité des formes de la subjectivité qui lui sont imposées (chef de famille, chef d'exploitation, ancien élève...), mais l'écriture personnelle introduit du jeu dans les postures attendues et les identités assignées.

Deuxièmement, s'y opère une condensation de formats d'écriture et de modèles variés (imprimés, manuscrits ; professionnels, scolaires, administratifs) sur un support unique, qui a cette caractéristique d'être à soi. Tension donc entre hétérogénéité des notations puisant à des sources diverses et unité du support personnel sur lesquelles elles sont recueillies.

Contrôler à son tour : reprises et déplacements 
Si l'appropriation du cahier en tant qu'objet en change l'usage, suspendant l'horizon du contrôle ou de l'inspection, les cahiers n'en puisent pas moins à une culture écrite où modèles et formats d'écriture sont très contraints.

L'écriture peut servir à reprendre pour son propre compte des modèles imposés, l'autorité de l'écrit étant réinvestie par le scripteur, comme dans le « contrat » de Demba analysé plus haut. Il s'agit là de retourner la modalité écrite du contrôle envers d'autres. Plus généralement, la consignation de transactions ou le relevé des quantités de coton récoltées dans la famille donnent lieu à des séries d'inscriptions qui permettent de s'assurer un contrôle sur les biens et les personnes. Moussa Coulibaly intitule ainsi « cahier de contrôle » plusieurs de ses cahiers, qui contiennent les crédits consentis dans le cadre de son activité de petit commerçant. La forme du contrôle écrit est aujourd'hui omniprésente dans des contextes domestiques (relevé pluviométrique, répartition du coton récolté par chaque membre de la famille), au sein de groupes de pairs (travail aux champs des membres d'une association de jeunes) et de relations de voisinage (partage de la viande lors de l'abattage d'une bête au sein d'un quartier). L'écriture du cahier offre un espace où éprouver une maitrise relative des relations aux autres et aux institutions.

Cet usage du cahier comme instrument de contrôle reflète un des premiers usages personnels de l'écrit : écrire, ou faire écrire, pour se prémunir contre d'autres écrits. Ainsi, conserver une contrepartie des écrits de l'encadrement agricole est une pratique courante qui n'a pas le cahier comme support exclusif. Lors de la pesée du coton, des bouts de papier soigneusement gardés par-devers soi visent à s'assurer que le montant publiquement annoncé lors de la pesée ne sera pas ensuite modifié. Ces procédés rappellent que dans une situation où l'alphabétisation demeure limitée, la maîtrise de l'écrit génère de nouveaux partages. La création des associations villageoises est souvent rapportée, dans les récits des acteurs de l'époque, à la volonté des villageois d'échapper aux malversations des agents de la CMDT 
lors de la pesée du coton. Mais la défiance reste de mise envers les villageois lettrés, soupçonnés eux aussi de manipuler l'écrit à leur avantage.

Ces usages privés de l'écrit peuvent inciter à penser l'appropriation de l'écrit comme un prolongement des injonctions à écrire : si le carnet d'exploitation n'a pas été diffusé tel quel, les cahiers en constitueraient en somme la forme aboutie, dans l'intériorisation des normes d'organisation sociale et de production agricole par les villageois lettrés. Cette lecture ne rend toutefois pas justice à la complexité de la pratique : les scripteurs de cahiers ne se contentent pas de reprendre les identités assignées par les institutions (chef de famille, responsable d'exploitation) et les tâches de contrôle afférentes, ils les remodèlent parfois à leur manière. Ainsi, l'échelle de la chronique familiale ne coïncide pas toujours avec la famille telle qu'elle est reconnue par l'administration et qu'objective le carnet de famille, base du calcul de l'impôt: on a vu que Makan Camara recense des naissances des épouses de son frère, reconstituant ainsi l'unité de la concession $(d u)$, pourtant scindée en deux exploitations correspondant à deux familles pourvue chacune de son carnet de famille. Noms et dates y sont traduits dans des référents plus familiers.

Surtout, l'écrit, en particulier depuis l'extension de la scolarisation, est distribué selon des lignes qui ne coïncident pas toujours avec les formes socialement reconnues de l'autorité. Certains écarts donnent lieu à une redistribution des responsabilités admise socialement, comme ces cadets qui, mettant leurs compétences lettrées au service de la famille, s’y voit reconnue une place nouvelle. Mais d'autres compétences ne sont pas sollicitées, tant leur usage par la famille est impensable : c'est le cas des femmes, dont les pratiques sont le plus souvent confinées à des domaines strictement personnels, et qui ne prennent généralement pas part aux écritures domestiques, telles la consignation des naissances. Dans certains cas, cultivées pour elles-mêmes, ces compétences non reconnues trouvent dans le cahier à soi un 
espace de repli et d'exercice, notamment à travers des pratiques de recueils (recettes médicinales en particulier).

\section{Enjeux du recueil : compiler et rassembler}

Présentant leurs cahiers, les scripteurs en donnent souvent des descriptions très homogénéisantes, faisant ressortir ce qui est le thème dominant de l'écriture. D'autres scripteurs s'excusent de leur hétérogénéité.

AM Les dates des semis, vous notez ça dans quel document? MC Je possède un cahier [pour cela]. AM Quel genre de cahier? Un carnet d'exploitation? MC Bon, c'est un cahier, un cahier vierge tout simplement (...). C'est un cahier, comme ça. AM Et sur votre cahier, vous notez uniquement les dates des semis ou aussi d'autres choses ? MC Ah ! On y écrit bien d'autres choses, puisque nos cahiers ne sont pas vraiment des cahiers dignes de ce nom ${ }^{60}$, on y écrit autre chose... (entretien traduit du bambara, interventions de l'assistant omises).

Cette hétérogénéité peut être réduite par des modalités d'écriture spécifiques qui constituent des espaces homogènes à l'échelle de la page, du cahier, ou de la pluralité des cahiers détenus par un scripteur qui leur assigne des domaines d'écriture distincts. Elle n'en caractérise pas moins les cahiers comme espaces de recueil.

Avant de nous arrêter sur la signification du recueil en un espace unique de notations diverses, un repérage des différentes sources auxquelles puisent les scripteurs est indispensable. Cellesci ne se réduisent pas à la consignation d'événements familiaux ou domestiques, ni même à la chronique, fût-elle élargie à des horizons lointains comme chez Makan. La copie de prières ou

\footnotetext{
${ }^{60}$ Cette expression vise à rendre l'emprunt au français utilisé en bambara « garantilenba », composé sur «garanti ».
} 
de manuels scolaires, la transcription d'incantations ou d'informations diffusées la radio (résultats sportifs ou titres de chansons) occupent une large part des cahiers.

Le recueil est un geste qui prend un relief particulier quand il s'agit de corps de savoir dont la transmission est contrôlée. En particulier, la notation de formules magiques, pratique récurrente dans les cahiers, instaure un rapport singulier à un domaine traditionnellement caractérisé par une transmission orale. Les scripteurs retravaillent ces contenus, en s'appuyant pour transcrire ces formules sur le modèle savant de la recette médicinale ${ }^{61}$. La notation de formules sur un cahier à soi, une des rares pratiques partagées par hommes et femmes, s'inscrit dans le contexte d'un recours de plus en plus individualisé aux savoirs magiques ${ }^{62}$. De manière similaire, consignant des faits d'histoire locale, le scripteur du cahier pose en chroniqueur ou mémorialiste, porteur d'une réputation familiale ou villageoise, sans y être nécessairement socialement autorisé, ce qui distingue ces chroniques des énonciations orales sur ces sujets, très contrôlées ${ }^{63}$. Enfin, les sélections qui s'opèrent, à travers la copie, au sein du corpus des textes sacrés de l'islam témoignent d'un rapport plus individualisé à ce savoir religieux que celui qui se transmet traditionnellement auprès d'un maître ${ }^{64}$.

Support de notations en apparence plus anodines telles que des informations entendues à la radio, le recueil, sélection singulière puisée à ces sources publiques, n'en a pas moins

\footnotetext{
${ }^{61}$ La recette est un des genres graphiques retenus par Jack Goody dans La raison graphique..., op. cit. chap. 7.

${ }^{62}$ Catherine BARRIÈRE, « Techniques d'agression magique en pays bamana (région de Segu, Mali) : emprunts réciproques entre islam et religion traditionnelle », Journal des africanistes, 69-1, 1999, p. 177-197, en particulier p. 182. Certaines pratiques magiques échappent à la mise en recette, notamment la fabrication des objets forts que sont les boliw, à propos desquels Jean Bazin rappelle : «Un boli n'est pas une préparation médicinale ou magique (fura, basi); il n'est pas définissable par une recette ; il a une identité qui peut être évoquée par des récits », Jean BAZIN, « Retour aux choses-dieux », in Charles Malamoud et Jean-Pierre Vernant (dir.), Corps des dieux, Paris, Gallimard, 1996, p. 253-273, ici p. 263.

${ }^{63}$ Pour une analyse des contraintes qui pèsent sur l'énonciation de choses tenues pour « secrètes », voir Jan JANSEn, Epopée, histoire, société : le Cas de Soundjata, Mali et Guinée, Paris, Karthala, 2003. Si la sphère que recouvre le cahier est souvent désignée par la scripteur par le recours à la notion de secret («gundo », en bambara), son contenu va bien au-delà du sens restreint de savoir dont la transmission est socialement contrôlée . Sur la polysémie la notion, voir Jan JANSEN et Molly ROTH (dir.), «Secrets and Lies in the Mande World », Mande Studies, ${ }^{\circ} 2,2000$.

${ }^{64}$ Cette pratique d'écriture s'inscrit dans une transformation plus globale du rapport au savoir islamique. Outre les brochures islamiques, d'autres médias jouent un rôle central dans la manière dont se forgent de nouveaux rapports à l'islam, voir Dorothea SCHULZ, «"Charisma and brotherhood" revisited: mass-mediated forms of spirituality in urban Mali », Journal of Religion in Africa, 2003, p. 146-171.
} 
d'importance. Il permet à son détenteur de prendre une place dans ces circulations culturelles en notant les résultats des compétitions sportives internationales, les titres des chansons qui passent à la radio, les adresses d'étrangers de passage, soit ce qui le rattache à un au-delà des sociabilités villageoises. Le scripteur intervient alors en tant qu'il se ressaisit de contenus qui circulent plus largement, mais dont le choix lui revient, selon des goûts qu'il trouve là l'occasion d'affirmer, et à propos desquels s'exprime un plaisir à écrire. Ici, la reprise de formes officielles intervient sur un mode différent de celui du «contrat» de Demba, celui du détournement ludique, comme cette demande de dédicace de chansons à la radio ornée d'un magnifique dessin de tampon entrelaçant les initiales du scripteur et le nom du village.

Cet aperçu des principales sources des cahiers et de la manière dont les scripteurs y puisent donne à voir la dimension expérimentale de cette pratique. Elle engage des actes d'écriture distincts $^{65}$ : copier, translittérer, transcrire de l'oral... L'emprunt de modèles graphiques va au-delà d'un simple transfert pour témoigner d'une recomposition des savoirs dont les scripteurs sont les acteurs.

\section{Privatisation et subjectivation}

On peut alors se demander jusqu'où la notion de «privatisation » saisit adéquatement les processus observés. Rapporté à la définition proposée pour l'Europe moderne («écart par rapport au public, au civil, aux affaires qui sont celles de la cité et de l'Etat ; écart par rapport à la famille, à la maison, aux sociabilités qui sont celles de l'intimité domestique ») ${ }^{66}$, le processus analysé ici pourrait sembler inabouti. Réinscrit dans un contexte où le récit de soi, même à l'oral, n'a rien d'évident, il prend sens par rapport à une diversité d'injonctions

\footnotetext{
${ }^{65}$ La notion d'actes d'écriture est reprise à Béatrice Fraenkel, qui en propose une élaboration théorique dans B. FRAENKEL, «Actes d'écriture : quand écrire c'est faire », Langage et société, n ${ }^{\circ} 121-122$-sept.-déc., 2007, p. 101-112.

${ }^{66}$ Roger CHARTIER, «Les pratiques de l'écrit », op. cit. ici p. 138.
} 
auxquelles les scripteurs se plient tout en les remodelant, y trouvant l'occasion d'expérimenter de nouvelles manières de se rapporter à soi ${ }^{67}$.

La notion de privatisation décrit bien à la fois le statut spécifique du support comme objet à soi, le geste de reprise de modèles pour son propre compte, et sa dimension constitutive. Le repérage des sources auxquelles puisent les cahiers montre que le recueil ne reconduit pas une familiarité déjà donnée, pas plus qu'il ne se limite à la reprise de contenus qui circulent globalement: il est le lieu d'une recomposition des savoirs complexe, médiatisée par le recours à des formes publiques. Cette expérimentation sur les formes et les contenus se fait dans un va-et-vient entre les différents espaces, sociaux et culturels, dans lesquels s'inscrit le scripteur.

L'analyse des cahiers permet d'aller plus loin, et d'identifier la mise à l'épreuve de ces différentes échelles comme centrale. Saisie dans ses hésitations mêmes, la tenue d'un cahier personnel rend compte du fait que l'échelle du privé ou du domestique circonscrite par les institutions ne va pas de soi, et qu'elle est sans cesse déplacée, vers des pratiques plus larges ou plus restreintes. Le terme de privatisation ne doit pas réduire cette oscillation: les notations concernant l'individu en propre sont étroitement mêlées à des notations domestiques ; les données compilées puisent à des savoirs partagés. Mais les modalités de leur rassemblement et de leur conservation sur un support à soi sont neuves, et ouvrent un espace d'expérimentation inédit.

\section{Conclusion}

Dans la région cotonnière du Mali, l'omniprésence de la culture écrite, au-delà d'une familiarité ancienne mais diffuse avec la tradition lettrée en arabe, est liée à la multiplication

\footnotetext{
${ }^{67}$ Voir Bogumil JEWSIEWICKI (dir.), Naître et mourir au Zä̈re. Un demi-siècle d'histoire au quotidien, Paris, Karthala, 1993. Dipesh Chakrabarty indique que l'appropriation des genres occidentaux d'écriture de soi dans l'Inde coloniale et postcoloniale ne donne pas lieu au repli sur une intériorité psychologique, voir

« Postcoloniality and the Artifice of History: Who Speaks for "Indian" Pasts? », Representations, 37, 1992, p. 126.
} 
des procédures d'enregistrement et d'inscription des individus, des familles et des propriétés, dans un contexte de fort encadrement agricole. Des attentes spécifiques ont été développées envers certaines catégories d'individus, notamment les premiers bénéficiaires de l'alphabétisation pour adultes en bambara dans les années 1970, souvent chefs d'exploitation. Enfin, la diffusion de l'écrit par la scolarisation a contribué au développement d'une génération de lettrés, écrivant à des degrés divers en français et en bambara, à partir du milieu des années 1980. Certains ont été sollicités pour prendre la relève des premiers alphabétisés au sein du bureau des associations villageoises, tandis que d'autres trouvaient à exercer leurs compétences dans des domaines privés.

Commune à ces différents lettrés, la pratique de tenir un cahier à soi prend des formes variées, mais se caractérise a minima par le caractère personnel l'objet, par décision initiale ou réemploi d'un cahier de formation. Elle donne un contenu à l'appropriation de l'écriture identifiée par de précédents travaux comme un trait central des sociétés, mêmes rurales, du Mali contemporain ${ }^{68}$

Reposant sur la reprise d'identités assignées et de modèles imposés, semblant parfois coller au plus près des modes d'inscription et d'enregistrement de l'administration, de la CMDT ou de l'école, le processus de privatisation n'est pas de l'ordre du repli sur un soi constitué en intériorité psychologique. Pourtant, la pratique d'écriture personnelle introduit des déplacements décisifs et constitue un espace nouveau.

Ecrire sur un support durable et personnel permet de contrôler les procédures écrites qui confèrent l'autorité, de les utiliser dans des occasions où personne ne les requiert, voire de les retourner en sa faveur pour s'assurer à son tour un contrôle sur d'autres. L'écriture personnelle tout à la fois cristallise les contours du privé et du domestique tels qu'ils sont reconnus par les institutions, et permet de définir des espaces sociaux de référence qui ne

\footnotetext{
${ }^{68}$ Voir Étienne GERARD, La tentation du savoir en Afrique... op. cit.
} 
coïncident pas exactement avec ceux-ci. Elle fournit un lieu où articuler ces différentes identités, ouvrant la voie à l'usage du cahier comme technique de soi, dans des investissements parfois intenses dans l'écriture.

L'étude d'une pratique partagée, par rapport aux travaux actuels qui prennent principalement la forme d'études de cas, permet de situer ces modalités diverses d'appropriation de l'écriture au sein d'un environnement graphique plus vaste. L'articulation entre les différentes strates de la culture écrite, en particulier la plurigraphie associant l'arabe, reste toutefois à approfondir ${ }^{69}$. L'approche par le support, méthode éprouvée sur d'autres terrains, apparaît extrêmement féconde pour saisir cet objet. On peut attendre du développement des travaux sur la culture écrite en Afrique l'émergence d'études de la culture matérielle de l'écrit associée aux modes de documentation de soi : si les recherches disponibles font ressortir l'importance du cahier et la proximité des modalités de l'archivage personnel, du sac plastique à la malle en fer-blanc, la description de ces pratiques et leur articulation avec l'émergence de nouvelles notions de la propriété restent à approfondir. Rejoignant des intérêts classiques de l'anthropologie pour la culture matérielle et l'organisation domestique, de telles études permettraient de prolonger la discussion engagée ici sur la notion de privatisation.

\author{
Aïssatou Mbodj-Pouye
}

Centre d'études africaines, EHESS

\footnotetext{
${ }^{69}$ Il s'agit là d'une des limites de l'ouvrage de Karin Barber, la culture lettrée en arabe n'apparaissant que de manière marginale (ce qui est lié aux terrains explorés). Voir Karin BARBER (dir.), Africa's Hidden Histories..., op. cit.
} 